\title{
Modeling Protein-Ligand Interactions with Graph Convolutional Networks for Interpretable Pharmaceutical Discovery
}

\section{Paola Ruiz Puentes}

Universidad de Los Andes

Laura Rueda-Gensini

Universidad de Los Andes

Natalia Valderrama

Universidad de Los Andes

Isabela Hernández

Universidad de Los Andes

Cristina González

Universidad de Los Andes

Laura Daza

Universidad de Los Andes

Carolina Muñoz-Camargo

Universidad de Los Andes

Juan C Cruz

Universidad de Los Andes

Pablo Arbelaéz ( $\nabla$ pa.arbelaez@uniandes.edu.co)

Universidad de Los Andes

\section{Research Article}

Keywords: Drug Discovery, Protein Ligand Interaction, Deep Graph Convolutional Networks, Adversarial examples.

Posted Date: February 2nd, 2022

DOI: https://doi.org/10.21203/rs.3.rs-1262123/v1

License: (c) (1) This work is licensed under a Creative Commons Attribution 4.0 International License.

Read Full License 


\title{
Modeling Protein-Ligand Interactions with Graph Convolutional Networks for Interpretable Pharmaceutical Discovery
}

\author{
Paola Ruiz Puentes ${ }^{1,2+}$, Laura Rueda-Gensini ${ }^{1,2+}$, Natalia Valderrama ${ }^{1,2+}$, Isabela Hernandez ${ }^{1,2}$, Cristina \\ González $^{1,2}$, Laura Daza ${ }^{1,2}$, Carolina Muñoz-Camargo ${ }^{2}$, Juan C Cruz ${ }^{2}$, Pablo Arbeláez ${ }^{*, 1,2}$ \\ ${ }^{1}$ Center for Research and Formation in Artificial Intelligence, Universidad de los Andes, Bogotá 111711, \\ Colombia \\ ${ }^{2}$ Department of Biomedical Engineering, Universidad de los Andes, Bogotá 111711, Colombia \\ + These authors contributed equally.
}

\begin{abstract}
Drug Discovery is an active research area that demands great investments and generates low returns due to its inherent complexity and great costs. To identify potential therapeutic candidates more effectively, we propose Protein-Ligand with Adversarial augmentations Network (PLA-Net), a Deep Learning-based approach to predict Protein-Ligand Interactions (PLI). PLA-Net consists of a two-module Deep Graph Convolutional Network that considers ligands' and targets' most relevant chemical information, successfully combining them to find their binding capability. Moreover, we generate adversarial data augmentations that preserve relevant biological backgrounds and improve the interpretability of our model, highlighting the relevant substructures of the ligands reported to interact with the protein targets. Our experiments demonstrate that the joint Ligand-Target information and the adversarial augmentations significantly increase the interaction prediction performance. PLA-Net achieves $86.52 \%$ in mean Average Precision for 102 target proteins with perfect performance for 30 of them, in a curated version of Actives as Decoys (AD) dataset. Lastly, we accurately predict pharmacologically-relevant molecules when screening the ligands of ChEMBL and Drug Repurposing Hub datasets with the perfect-scoring targets.
\end{abstract}

Index Terms-Drug Discovery, Protein Ligand Interaction, Deep Graph Convolutional Networks, Adversarial examples.

\section{INTRODUCTION}

The development of novel drugs with therapeutic potential is a challenging yet essential endeavor to ensure human welfare and actively confront health threats. This process is characterized by conventional cycles lasting up to 12 years and demanding costs of about 2.7 billion US dollars [1], with declining compensation due to limited efficacy and safety issues during clinical trial stages [2]. In this regard, the likelihood of approval for small molecule candidates for the period 20112020 was only $7.5 \%$ [3], with expenditures ranging from 800 million to 1.4 billion US dollars in unsuccessful clinical trials [4]. These large yet unfruitful investments are currently forcing pharmaceutical and related sectors to search for more efficient strategies in terms of research objectives and profitability.

To address these shortcomings, reverse pharmacology proposes more robust data-driven approaches to improve the identification of active molecules towards therapeutic biological targets [5]. This approach's main promise is the possibility to increase predictability and accuracy of drug screening processes by systematizing analyses over large pharmaceutical datasets, which results in lessening the need for laborious in vitro and in vivo experimentation, and a significant increase in the overall efficiency of the entire discovery process. In this regard, virtual screening emerges as the current standard for the prediction of interactions between small molecules and proteins [6]. Conventional methods in virtual screening mainly employ molecular docking techniques, but are still limited in accuracy and effectiveness, and often require expensive experimental testing for validation prior to market launching [7], [8]. In consequence, there is still a large scope for improving screening processes, especially regarding biological compatibility prediction.

Recent works use Deep Learning (DL) techniques in broad applications of the molecular biology domain, whose understanding is critical for the development of medicine and the comprehension of the biological interactions during physiological processes. For example, the prediction of functions for programmable RNA switches [9], [10], the three-dimensional structure prediction of proteins [11], [12], the prediction between protein-protein interfaces [13], and the discovery of structurally distinct novel antibiotics [14] are tasks in which the correct approach to the problem and data modeling allows DL to carry out the intended task successfully. Consequently, it is possible to reduce the laboratory experimentation or even replace it, especially when it is not possible to study it experimentally. Likewise the research topics mentioned above, the interaction between small molecules and proteins is essential in the development of medicine, and therefore, in human health. The information provided by the analyses of these interactions, such as the functional units, the binding pockets, and interaction sites between drugs and targets, is crucial to the targeted development and design of pharmaceuticals. For this reason, it is of general interest to know both if there is a protein-ligand interaction and how this interaction occurs.

In this perspective, deep learning methods might be able to play a pivotal role due to their ability to find and exploit 


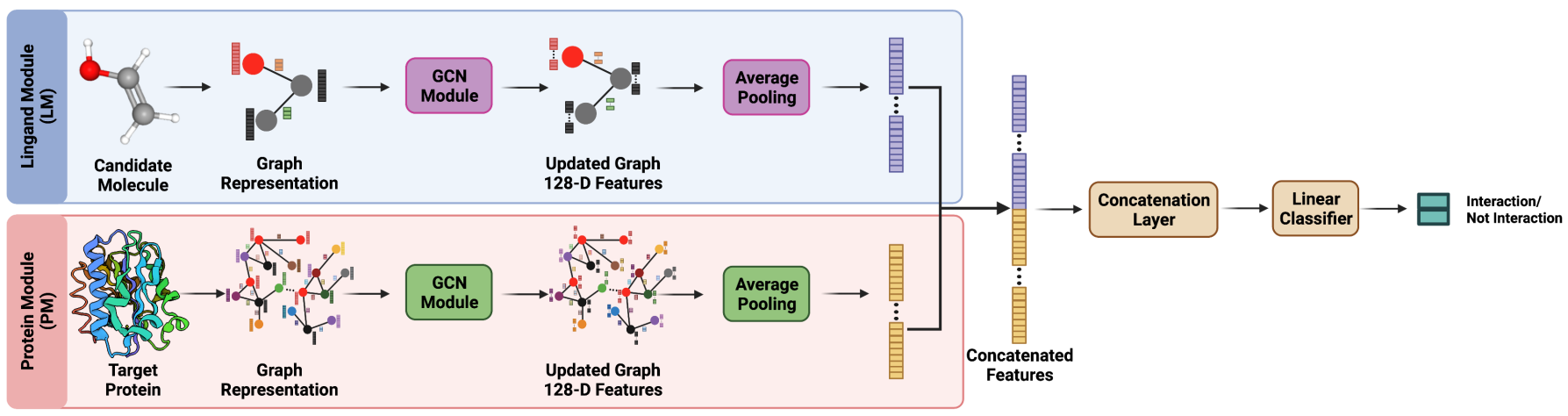

Fig. 1: PLA-Net Workflow. Molecule and protein graph representations are generated from SMILES and FASTA sequences and used as input to the Ligand Module (LM) and Protein Module (PM), respectively. Each module comprises a deep GCN followed by an average pooling layer, which extract relevant features of their corresponding input graph. Both representations are finally concatenated and combined through a fully connected layer to predict the Protein-Ligand Interaction probability.

patterns in large datasets and distill salient features that characterize effective biological interactions between cellular targets and small molecules, henceforth regarded as Protein-Ligand Interactions (PLIs) [15]. Therefore, we address one of the most common formulations for PLI understanding: the binary classification of active and non-active interactions between a ligand and a protein. This task substantiates the larger endeavor of the niche market for drug discovery and repurposing, whose size is projected to grow from 24.96 to 34.62 billion US dollars between 2020 and 2027 [16].

Understanding biological interactions of pharmacological molecules involves reasoning over their intricate structures and corresponding traits at the atomic level. This working objective stresses the importance of appropriate molecular structure representation. In this respect, previous approaches have employed two-dimensional molecular images built from one-hot embeddings over atoms, and have been analyzed under Convolutional Neural Networks (CNN) [17] and Recurrent Neural Networks (RNN) [18] to predict PLIs. However, these contributions disregard molecular structure, as well as the target protein's information. Alternatively, [19] and [20] incorporate the 3-dimensional structural information of the ligand positioned in the target receptor to predict activity through CNNs. Nonetheless, this type of input complex needs additional pre-processing and previous knowledge of the 3-D structure of the protein, its binding pockets and the ligand position within the pocket, which are not easily available. Given the non-Euclidean nature of chemical data, molecular graph representations enable a more accurate and explicit modeling of atom and bond spatial configurations. Moreover, the conjunction of graph representation and deep learning techniques provides a promising approach to model molecular structures, both of small molecules and proteins, while extracting features with relevant biological backgrounds [21], [22].

Despite recent success in improving PLI predictions, their interpretability is a recurrent issue to validate their translation into compelling medicine scenarios. This feature is particularly important since high-performance metrics fail to provide sufficient information for evaluating if the model is learning relevant biological and chemical data for pharmacological design. To circumvent this situation, attention methods have emerged as powerful tools to identify key substructures in both targets and ligands for the model's predictions [22]. Zheng et al., for instance, implemented a multi-head attention algorithm for both target and ligand embeddings to visualize more easily where the model focuses to predict PLIs [23]. Even though their method is able to identify real overlapping regions of interaction in both ligands and targets, they disregard structural information by using linear representations of the molecules (i.e., SMILES strings).

In this work, we propose the application of Graph Convolutional Networks (GCNs) for predicting PLIs where graphbased representations of both molecules and target proteins are obtained from easily accessible SMILES and FASTA sequences. We address the most common limitations of GCNs, such as depth limits due to vanishing gradients, oversmoothing and loss of spatial information, by building on the work of [24] and [25] to the context of PLIs. We harness their proposed method for extrapolating common CNN strategies (e.g., residual and dense connections, dilated convolutions) to GCNs, which they demonstrated capable of enabling the training of deeper networks for a variety of computer vision tasks. Moreover, we propose a method for increasing the interpretability of our PLI predictions by training the networks with adversarially-augmented molecules. These, in turn, are inspired by adversarial examples, which have gained increasing popularity over the past few years in the field of computer vision due to their ability to direct learning towards semantically-aligned features. In particular, we introduce a biologically-bounded gradient-based method to generate adversarial molecule augmentations, which adapts gradient-based edge deletion methods previously proposed for adversarial examples in graph data [26]. This is critical to preserve biological consistency and essential class features in molecular graphs.

Our network, henceforth termed Protein-Ligand with Adversarial augmentations Network (PLA-Net), comprises two modules that separately extract chemical and structural information from proteins and ligands, thereby learning optimal 
representations for further compatibility analysis (Figure 1). Both representations are then correlated through a linear layer. Accordingly, our contributions are three-fold: (i) we model the spatial configuration of both the target protein and the ligand through bidirected graphs, (ii) we integrate the structural information of proteins for PLI prediction, and (iii) we propose a gradient-based method to compute adversarial molecule augmentations that preserve relevant biological backgrounds and improve both interpretability and overall performance. Additionally, we establish the new state-of-theart, outperforming the previous one [18] by a large margin of $19.8 \%$ on mAP in a curated version of the AD Dataset. Both the outstanding performance and the interpretability results position PLA-Net as a promising starting point to integrate deep learning methods into drug discovery pipelines.

To support the previous statement, we explore the main contributions of our work in the reported literature. First, we perform virtual screening between the ligands of two large datasets (ChEMBL [27], and Drug Repurposing Hub [28]) with the perfect-scoring targets, finding that our method predicts as active experimentally validated molecules. Second, PLA-Net can provide insight into the relevant substructures that may interact with proteins of clinical interest, even if their 3D structure is not available; enabled by the increased interpretability and the simple input needed for the model, a FASTA and a SMILES sequence. This information makes it possible to optimize or design pharmacological molecules that have the structure predicted to interact with a specific target. Finally, PLA-Net addresses the much-needed increase in effectiveness, for the identification and production of therapeutics with superior selectivity and efficacy.

\section{PLA-Net FramewORK}

PLI Benchmark. The Database of Useful Decoys Enhanced (DUD-E) is widely employed to benchmark approaches that predict PLIs [29], [30], [31]. DUD-E contains 22,886 experimentally verified active compounds towards 102 proteins. Some of these proteins are related to chronic diseases of significant clinical interest such as hypertension (RENI), HIV (CXCR4), cancer (FPPS), and Parkinson (COMT). Additionally, for each active molecule, DUD-E contains 50 non-active compounds (decoys) with similar physicochemical properties but different topologies. This unbalanced data distribution replicates the real scenario of finding an active compound against a huge variety of decoys.

However, Chen et al. [32] proved that DUD-E's selection criteria for the decoys follows a pattern that makes them easily distinguishable from the active molecules. Conversely, they proposed the Actives as Decoys (AD) dataset, in which the decoys are selected from the active molecules of other protein targets. In particular, the decoys for a protein are selected by performing molecular docking between that protein and the active compounds from the other 101 proteins. The PLI is then ranked based on the predicted binding energy. The top50 molecules of each of the 101 targets are selected to be the decoys of that target protein. This process is repeated for all targets to create the decoy dataset.
Nonetheless, we noticed that some molecules in the DUD-E dataset are labeled as active for more than one protein target and, therefore, the decoy selection process of the AD dataset causes some molecules to be simultaneously labeled as active and decoy for the same protein. In addition, most decoys for the same protein are repeated. Since this contradictory and redundant information might detrimentally lead to a bias during model training, we removed the repeated samples $(546,412$ molecules) and variably labeled samples (37 molecules) for each target, which represent $52.29 \%$ of the original data. We also ensure that active compounds of the targets are the same as those experimentally validated in DUD-E. Subsequently, we separated the curated version of the AD dataset into training and testing sets, comprised of $90 \%$ and $10 \%$ of the compounds for each protein, respectively. Next, we performed a four-fold cross-validation with the training subset. We also included the structural information of each protein by compiling the FASTA sequence for each of the 102 targets from the Universal Protein (UniProt) repository.

We evaluated with the Average Precision (AP) metric and report the mean AP (mAP) for all the 102 target proteins.

Molecule representation. The molecular representation used by state-of-the-art methods in the AD Dataset, SMILES, fails to recapitulate the complexity of molecules mainly due to the absence of structural information that dictates interactions between atoms. Other methods rely on very complex 3D representations, which cannot be obtained for all proteinligand pairs because they depend on experimental techniques such as X-ray crystallography and computational techniques such as molecular docking. In contrast, graph representations are well suited for capturing molecular information since they enable the reconstruction of atomic networks that preserve chemical and structural information. For this reason, we built the graph representations of PLA-Net based on SMILES and FASTA sequences to exploit the information from both ligands and target proteins, respectively (Figure 1). Given a molecule, its graph is represented as $\mathcal{G}=\left(\mathcal{V}, \mathcal{E}, X_{v}, X_{e}\right)$, where $\mathcal{V}$ denotes the set of atoms (nodes), $\mathcal{E}$ the set of bonds (edges), $X_{v}$ the set of atom features and $X_{e}$ the set of bond features. An atom feature vector $x \in X_{v}$ comprises nine properties of the atom $v \in \mathcal{V}$ : atomic number, chirality, degree, formal charge, number of hydrogens, number of radical electrons, hybridization, aromaticity and ring membership. Similarly, we built a bond feature vector $x_{v u} \in X_{e}$ from three characteristics of the bond $e_{v u} \in \mathcal{E}$ between atom $v$ and atom $u$ : type of bond, stereochemistry, and conjugation. Each feature is represented as a one-hot vector and all one-hot feature representations are then concatenated to form the feature vector. The length of each one-hot vector is determined by the possible options for describing each feature, which are summarized in Table 1. Moreover, all bonds are assumed bidirectional, $X_{e_{v u}}=X_{e_{u v}}$. Accordingly, this graph representation enables the learning of both chemical and spatial distribution properties due to the node/edge feature vectors and edge configurations between nodes, respectively. Furthermore, this representation favors the learning of implicit 3D information that is inherent to the modeled molecules such as the stereochemistry of bonds, 
TABLE I: Atom and bond features. Feature vectors are obtain using RdKit and OGB libraries, which describe the state of an atom and a bond within a molecules.

\begin{tabular}{c|c}
\hline \hline \multicolumn{2}{c}{ Atom Features } \\
\hline \hline Atomic Number & $1,2, \ldots, 119$ \\
\hline Chirality & $\begin{array}{c}\text { Unspecified, Tetrahedral clockwise, } \\
\text { Tetrahedral anti-clockwise, Other }\end{array}$ \\
\hline Degree & $0,1, \ldots, 10$ \\
\hline Formal Charge & $-5,-4, \ldots, 4,5$ \\
\hline Number of Hydrogens & $0,1, \ldots, 8$ \\
\hline Number of radical $\mathrm{e}^{-}$ & $0,1, \ldots, 4$ \\
\hline Hybridization & $\mathrm{Sp}_{\mathrm{Sp}}{ }^{2}, \mathrm{Sp}^{3}, \mathrm{Sp}^{3} \mathrm{~d}, \mathrm{Sp}^{3} \mathrm{~d}^{2}$ \\
\hline Aromaticity & 0,1 \\
\hline Ring membership & 0,1 \\
\hline \hline \multicolumn{2}{c}{ Bond Features } \\
\hline \hline Type & Single, Double, Triple, Aromatic \\
\hline Stereochemistry & None, Z, E, CIS, TRANS, Any \\
\hline Conjugation & 0,1 \\
\hline
\end{tabular}

chirality, and aromaticity.

Architecture details. As shown in Figure 1, ligand and protein graphs are analyzed separately by a GCN module, which extracts structural and spatial information to obtain optimal representations for further PLI analysis. Each module comprises a deep GCN and an average pooling function, which outputs a 128-D vector representation for the molecular graph. Both optimized representations are concatenated, a linear layer merges the information, and a fully connected layer then classifies the ligand as an active or decoy.

Our GCN modules were adapted from [25], which is a message-passing framework originally designed for molecular property prediction. Being $\mathcal{N}_{v}$ the set of neighbors of atom $v$, the message passing algorithm of the l-th layer is described by message construction (Eq. 1), message aggregation (Eq. 2) and node update (Eq. 3) functions.

$$
\mathcal{M}_{v u}^{(l)}=\rho^{(l)}\left(X_{v}^{(l)}, X_{u}^{(l)}, X e_{v u}^{(l)}\right), u \in \mathcal{N}_{v}
$$

$$
\mathcal{M}_{v}^{(l)}=\zeta^{(l)}\left(\left\{\mathcal{M}_{v u}^{(l)} \mid u \in \mathcal{N}_{v}\right\}\right)
$$

$$
X_{v}{ }^{(l+1)}=\phi^{(l)}\left(X_{v}{ }^{(l)}, \mathcal{M}_{v}^{(l)}\right)
$$

where $\rho^{(l)}, \zeta^{(l)}, \phi^{(l)}$ are all learnable and differentiable functions. The message construction function $\rho^{(l)}$ is applied to the features of atom $v$, the features of its neighbor $u$, and their corresponding edge features $x_{v u}$ to obtain an individual message $\mathcal{M}_{v u}^{(l)}$ for each neighbor $u \in \mathcal{N}_{v}$. The message aggregation function $\zeta^{(l)}$ takes as input the set of individual messages and outputs an aggregated message. In this case, $\zeta^{(l)}$ is a learnable softmax function. Finally, $\phi^{(l)}$ updates the node features of the l-th layer by adding the aggregated message $\mathcal{M}_{v}^{(l)}$ and passing it through a multi-layer perceptron.

As mentioned in [24] and [25], to enable the adequate training of deep GCNs this architecture employs two techniques inspired in CNNs: (i) pre-activation variants of residual connections, and (ii) dilated aggregations that enlarge the receptive field. In the former, training proceeds by a change in the usual ordering of GCN components. In other words, instead of performing the graph convolution, followed by normalization, a ReLU layer and the addition of the residual connection, this architecture performs first the normalization and ReLU activation, followed by the graph convolution and the addition of the residual connection. In the latter, inspired by dilated convolutions in CNNs, a dilated graph is constructed after each message passing layer [33]. In particular, the network relies on a Dilated $k-N N$ to find dilated neighbors at a $d$ dilation rate. This operation returns the $k$ nearest neighbors within the $k x d$ neighborhood region by skipping every $d$ neighbors.

Our modules consist of 20 message-passing layers, and final embedding size for nodes' and edges' features of 128 .

Adversarial Data Augmentations. To generate biologically relevant adversarial molecules, we propose a gradient-based method that modifies the edges of molecular graphs according to their contribution to the model's outcome. In particular, we associate a binary coefficient with a value of 1 to each edge in the graph and multiply it with the feature vector of its corresponding edge during graph construction. Even though this multiplication maintains the original values of the feature vector and the computation of the molecule's embeddings, the gradient of these binary variables after backpropagating through the model becomes crucial for determining which edges are contributing the most to the model's predictions. In this context, the work by [26] inspired us to delete the edge with the most negative gradient coefficient, that is, the one with the highest contribution in minimizing the loss function.

To ensure that the augmented molecule resulting from the deletion of the chosen edge preserves relevant class characteristics, we defined a molecular distance metric that bounds the edge selection process. We impose a distance constraint between the augmented molecule and the original molecule as a function of the similarity between the former and the BemisMurcko scaffold [34] of the latter. This scaffold preserves crucial structural characteristics of a molecule by retaining backbone structures and eliminating side-chain elements [34]. We chose this scaffold as reference, instead of the original molecule, since we wanted to preserve molecular backbones in the augmented molecules. This is particularly important since modifying these structures may induce large conformational changes that could detrimentally impact the PLI under real physiological scenarios and, in turn, yield molecules that fail to preserve relevant class characteristics. Moreover, considering that Bemis-Murcko scaffolds were previously used to eliminate similar molecules while assembling the DUD-E dataset [29], these scaffolds are highly suitable for molecule comparison.

The distance between augmented molecules and their respective scaffolds is computed over their Morgan fingerprints according to Equation 4.

$$
d\left(M_{s}, M^{\prime}\right)=1-R G S\left(M_{s}, M^{\prime}\right)
$$

where $M_{s}$ is the Morgan fingerprint of the Bemis-Murcko scaffold of the original molecule, $M^{\prime}$ is the Morgan fingerprint of the augmented molecule, and RGS is the RogotGoldberg similarity between them. RGS was chosen above other fingerprint similarity metrics (e.g., Tanimoto, Dice, Sokal, Russel) since it minimizes the average distance between 


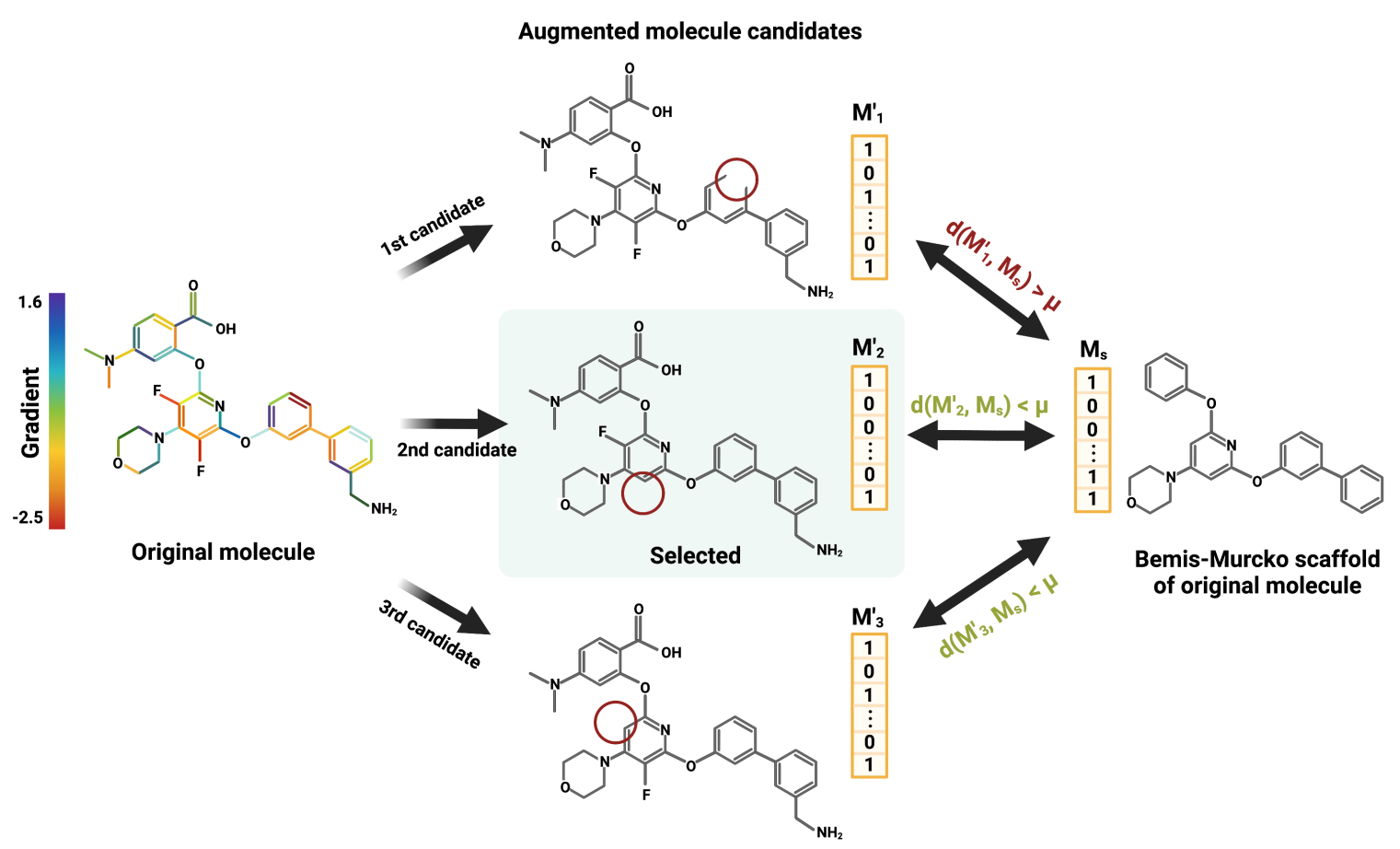

Fig. 2: Adversarial augmentation process. Augmented molecules are generated through an edge-deletion process that selects the edge of the molecular graph to delete by following two criteria: (i) the deletion of the selected edge must generate an adversarial molecule whose distance to the Bemis-Murcko scaffold of the original molecule is less than a defined threshold $(\mu)$ and (ii) the selected edge must have a negative gradient and the gradient magnitude must be maximal.

the fingerprints of molecules and their corresponding BemisMurcko scaffolds (Figure 3). To bound the described distance, we define the largest distance from the molecules to their corresponding scaffolds as a threshold $\mu$. If deletion of an edge causes the distance between the modified molecule and the scaffold of the original molecule to exceed the defined threshold, that edge is skipped to evaluate the next candidate, according to the negative gradient magnitude. If no edge with a negative gradient satisfies this condition, the molecule is skipped and no augmented molecule is retained. An example of this adversarial augmentation process is shown in Figure 2.

We generated augmented molecules for each batch and included them during training in addition to the original molecules. In this way, each batch included augmented molecules that exploit the strengths and weaknesses of the model at each training stage, tailoring the augmentation process to the model's needs.

Implementation details. PLA-Net is trained following a multi-step curriculum directed towards optimizing the molecule and protein representations such that their relevant information could be easily extracted and assembled. First, the Ligand Module (LM) was individually trained with only original molecules for 300 epochs and a learning rate (LR) of $5 e-3$. A randomly-initialized Protein Module (PM) was then included and jointly trained with the LM for 20 more epochs to integrate protein information. Simultaneously, the LM was trained from scratch with both original and augmented molecules for 300 epochs and a LR of $5 e-4$. Finally, the 128-

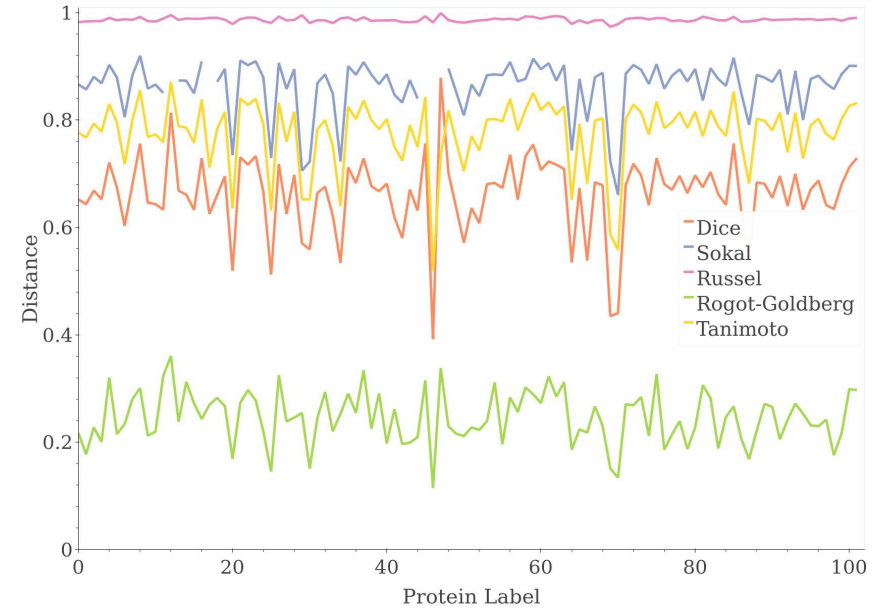

Fig. 3: Comparison of intra-class distance as a function of different similarity metrics. We computed the distances between molecules from a specific target class and their corresponding Bemis-Murcko scaffolds to assess the average intra-class distance as a function of different similarity metrics. We selected the Rogot-Goldberg similarity descriptor due to its high performance for intra-class similarities.

D feature embeddings generated by the PM jointly trained in the first stage, and by the augmented LM of the second stage were concatenated and their extracted information was combined with the training of a linear layer for 20 epochs and a LR of $5 e-5$. In this final step, the concatenated information 
TABLE II: Graph representation ablation study. LM: Ligand Module.

\begin{tabular}{ccc} 
Method & $\begin{array}{c}\text { Multiclass } \\
\text { benchmark }\end{array}$ & $\begin{array}{c}\text { Binary } \\
\text { benchmark }\end{array}$ \\
\hline \hline PharmaNet [18] & 66.7 & 72.56 \\
LM & 72.29 & 82.58 \\
\hline PLA-Net & - & $\mathbf{8 6 . 5 2}$
\end{tabular}

is first transformed into a 128-D vector with the defined linear layer and then passed through a classification layer that yields the binary prediction for the PLI.

Screening for Drug Discovery and Drug Repurposing. To validate the quality of our models' predictions and propose new pharmaceutical candidates for our perfect scoring targets, we screened for PLIs on the ChEMBL [27] and the Drug Repurposing Hub [28] databases. ChEMBL is a manually curated database with information for $15^{\prime} 504,604$ bioactive molecules with drug-like properties [27]. Similarly, the Drug Repurposing Hub is a curated and annotated collection with 13,553 FDA-approved drugs, clinical trial drugs, and preclinically tested compounds developed with the aim of revealing new therapeutic targets for known drugs [28].

We selected the models that achieved perfect PLI scores on the test set (i.e., models for 30 protein targets) for predicting PLIs with molecules in the Drug Repurposing Hub. Out of the selected models, we chose 11 to evaluate PLIs with ChEMBL molecules according to their clinical and therapeutic relevance. We filtered out from both databases the molecules whose SMILES could not be converted into a graph due to RDKit SMILES'-reading format. After this curation, we tested the 30 perfect-scoring models with 6,798 unique molecules of the Drug Repurposing Hub and the referred 11 models with 2'031,651 unique molecules of ChEMBL. Lastly, we selected the molecules with the 5 highest PLI scores for each tested target, after ensuring that those molecules were not considered for training the model.

\section{RESULTS AND DISCUSSION}

PLA-Net considerably outperforms State-of-the-art models in the proposed benchmark. Figure 4a shows that PLA-Net significantly improves PLI prediction and outperforms by a large margin PLI state-of-the-art methods [32], [18] and DeeperGCN [25] trained for this task. Besides increasing over 19 points in mean average precision (mAP) from the highest-performing method [18], the performance distribution of PLA-Net is superior than in the referred methods. Figure 4b shows that PLA-Net considerably shifts the performance histogram of the 102 models to the right, when compared to the current state-of-the-art method (PharmaNet) [18]. The models for 47 targets in PLA-Net achieve performances above $95 \%$ AP, which contrasts with only 18 targets in the case of PharmaNet. Moreover, 32 of these models achieve perfect scores, compared to only 13 in [18]. This proves that the method and training curriculum proposed enable a more explicit modeling of PLIs than
TABLE III: Ligand Module optimization. To optimize LM module, we performed ablations over 15 representative targets, which were selected according to varying levels of difficulty.

\begin{tabular}{|c|c|}
\hline Ligand Module Ablation & $\operatorname{mAP}(\%)$ \\
\hline \multicolumn{2}{|c|}{ Number of Message Passing Layers (Depth) } \\
\hline 7 Layers & 76.09 \\
\hline 15 Layers & 75.92 \\
\hline 20 Layers & 83.13 \\
\hline 50 Layers & 81.41 \\
\hline \multicolumn{2}{|c|}{ Aggregation Function } \\
\hline PowerMean Function & 77.95 \\
\hline Softmax Function & 83.13 \\
\hline \multicolumn{2}{|c|}{ Graph Pooling Method } \\
\hline Max & 77.95 \\
\hline Sum & 74.98 \\
\hline Mean & 83.13 \\
\hline \multicolumn{2}{|c|}{ Features Hidden Size } \\
\hline 64 & 62.08 \\
\hline 128 & 83.13 \\
\hline 256 & 82.46 \\
\hline
\end{tabular}

previous works.

Graph representations capture more relevant molecular information than linear representations. To exploit the information-rich graph representations proposed, we optimized the parameters of our LM (e.g., optimizer, scheduler, average pooling method, depth) to boost its discriminative power. $\mathrm{Ab}$ lations were performed over 15 representative targets, selected according to varying levels of difficulty are shown in Table III. We compared the performance of the optimized LM with the current state-of-the-art approach (i.e., PharmaNet) [18], which takes as input the SMILES strings for each molecule. Since PharmaNet was originally trained in a multiclass scenario, we trained our LM in the same setting and retrained PharmaNet in our proposed binary classification setting to compare their performance in both cases. We ablated the protein information from our model to ensure a fair comparison between methods.

Table II shows that our LM outperforms PharmaNet in both multiclass and binary setups by $5.59 \%$ and by $10.02 \%$, respectively. These results highlight the importance of structural information granted by graph representations of ligands, especially when training binary models for predicting PLIs with each target. Even though training one model is inherently more efficient, this training time is counteracted by the benefit of models achieving highly accurate predictions for specific therapeutic targets with clinical relevance. Accordingly, all experiments were performed in the binary setup.

Including protein information improves PLI predictions. Since the information of target proteins is fundamental when assessing a PLI, we hypothesized that its inclusion to enhance the classifier's discriminative power between actives and decoys. As expected, we show in Figure 5 that including protein information increases the performance of $66.7 \%$ of the 102 models and improves the mAP metric by 1.19 points 


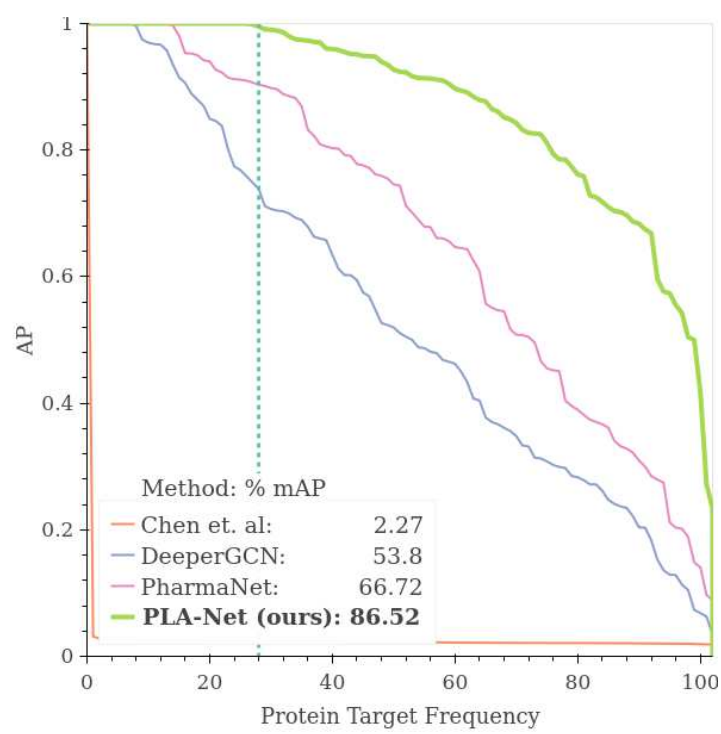

(a)

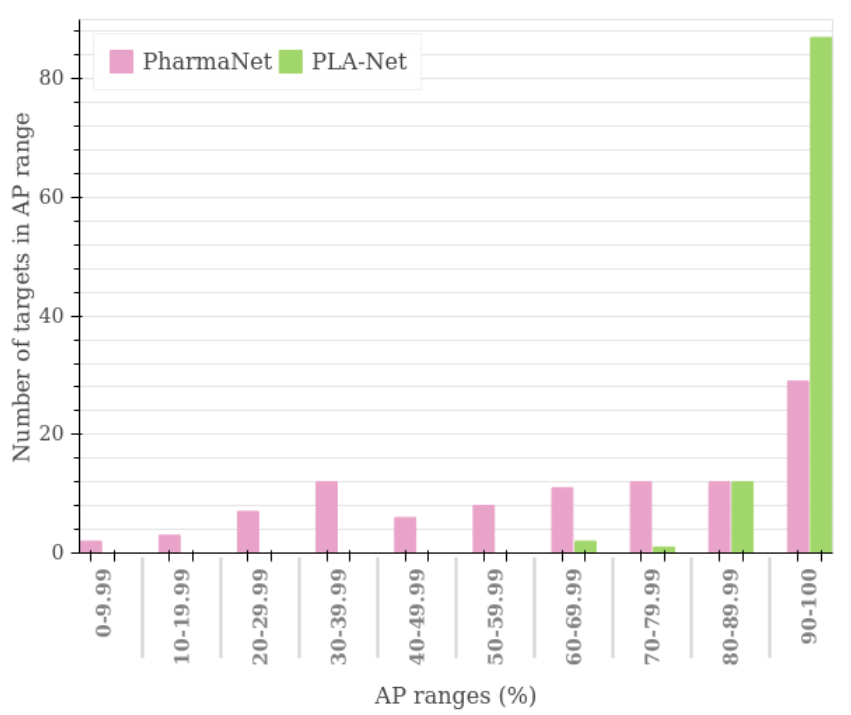

(b)

Fig. 4: Comparison with state-of-the-art methods trained for PLI prediction in the proposed benchmark. (a) Performance distribution curves comparing our model (PLA-Net) with state-of-the-art methods. For each model, we show the number of binary models that achieve a PLI prediction performance greater than or equal to a specific AP value. (b) We compare the performance distribution of the 102 targets in PLA-Net with that of the current state-of-the-art (PharmaNet) [18], showing that PLA-Net consistently improves the AP metric of the majority of the targets, with 87 targets with performance between 90-100\% vs. 29 in PharmaNet. Furthermore, PLA-Net achieves perfect performance for 30 targets with high clinical interest.



Fig. 5: Performance distribution of PLA-Net training stages. The performance of individual targets shows a marked tendency towards high and perfect mAP scores $(90-100 \%)$ as the training curriculum progresses. In particular, LM+PM and augmented $\mathrm{LM}(\mathrm{LM}+\mathrm{A})$ show a clear improvement in performance distribution with respect to LM, and this is further improved when combining the information extracted by each in PLA-Net. Best viewed in color.

when compared to the LM alone. The incorporation of target protein information during $\mathrm{LM}+\mathrm{PM}$ training was optimized by including a learnable parameter that multiplied the linear layer weights associated with the protein information, thus 


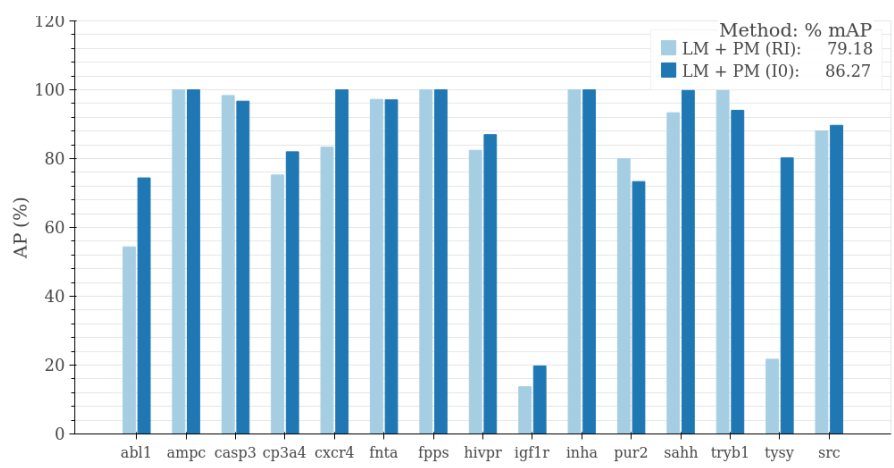

Fig. 6: Initialization of protein contribution during LM+PM training. Zeroing (I0) the linear classifier's weights that correspond to the protein information at the outset substantially improves the performance of the LM+PM with Random Initialization (RI). We measured performance in $\mathrm{mAP}$ for 15 representative targets.

controlling their contribution. We also show that initializing this multiplier as zero increases the performance of our models by allowing a smoother incorporation of this data when compared to random initializations (Figure 6). This ablation was performed over the models for the same 15 representative targets selected above and resulted in an increase of 7.08 points in the mAP metric with respect to randomly initialized weights.

Adversarial data augmentations improve model performance and interpretability. We propose the inclusion of adversarial molecule augmentations specifically tailored to harness the model's weaknesses during training. Accordingly, we intend to help the model learn semantically-aligned features of the active molecules for each target and effectively discriminate them from decoys. Following the augmentation process described in Section 2.4, we generated adversarial examples of the active molecules to each target at each training stage. This augmentation regime improved the average performance of the LMs alone by 3.25 points, increasing the performance of $55 \%$ of the 102 models and maintaining the performance of $40 \%$ (Figure 5). In particular, 13 of the models increased by more than 10 points in AP. This suggests that the generated active molecules in each training stage were sufficiently similar to experimentally-proven ligands to maintain class consistency but also sufficiently different for the model to learn relevant features that it failed to learn with the original dataset.

To assess the effect of the proposed training schemes over the interpretability of our model, we conducted a gradientbased analysis to highlight the learned molecular salient features in each scenario. The atoms with the lowest gradient within the molecular graphs of active molecules were interpreted as the most important for the PLI prediction, due to their highest contribution towards minimizing the loss function. To exemplify this analysis, Figure 7 compares the predicted importance of different molecular substructures when training with augmented molecules, with protein structural information or with neither. Notably, most models trained with either type of information exhibit marked attention shifts towards localized substructures that have been previously reported to be involved in the respective PLIs. For instance, adenine and ribose substructures are predicted to be the most important by $\mathrm{LM}+\mathrm{A}$ and $\mathrm{LM}+\mathrm{PM}$ models, respectively, for PLIs with the adenosine $\mathrm{A} 2$ receptor (AA2AR), while the pristine $\mathrm{LM}$ model fails to capture this information despite these substructures belonging to adenosine molecules that are known ligands for this target [35]. Similarly, the glutamate backbone in ligands for the glutamate ionotropic receptor (GRIK1) is partially or completely highlighted when employing either of these training schemes, while it is given the least importance when training without them. Considering that the pristine LM model for predicting PLIs with GRIK1 already achieves a perfect-score, this suggests that the additional information included during $\mathrm{LM}+\mathrm{A}$ and $\mathrm{LM}+\mathrm{PM}$ training is shifting model training towards learning interpretable features. This statement also holds true for targets whose ligands are more heterogeneous such as the adenosine deaminase (ADA), dihydrofolate reductase (DYR) and the epidermal growth factor receptor (EGFR), although much more prominently for adversarially-augmented models. In these cases, $\mathrm{LM}+\mathrm{A}$ is able to focus on relevant substructures whose interaction with the target's binding pocket has been previously described with molecular docking analyses (e.g., azole groups for ADA [36], 4-amino groups and pteridine rings for DYR [37] and quinilone N1 and nitrile groups for EGFR [38]). Moreover, the importance of specific functional groups directly involved in the function of enzymatic targets is also best captured by $\mathrm{LM}+\mathrm{A}$ models, such as carbonyl groups in the ligands for 11-b-hydroxysteroid dehydrogenase 1 (DHI1), which are reduced into hydroxyl groups upon their interaction with DHI1 [39].

However, the attention shifts induced by the inclusion of target protein information in some $\mathrm{LM}+\mathrm{PM}$ models was not as fruitful as when training with augmented molecules. Phenol groups in ligands for the estrogen receptor alfa (ESR1) [40], for example, lose importance in LM+PM models despite being directly implicated in the PLI of this ligand with its receptor. Moreover, the importance of the N2 and nitrile groups of quinilone [38] is completely lost when training with the protein information of EGFR, even though the AP achieved by the LM+PM model for this target is higher than that of the LM model. Overall, these results suggest that the observed increase in interpretability is not merely a result of the increase in model performance, but that the type of additional information does play a role in what the models are learning. Specifically, the additional information provided by the generated adversarial molecules appears to be particularly useful in learning semantically-aligned features.

PLA-Net's perfect scoring models predict pharmacologically-relevant PLIs on Drug Repurposing and ChEMBL databases. A high-throughput virtual screening for pharmacologically-relevant molecules on Drug Repurposing and ChEMBL databases revealed highly accurate predictions for PLIs with 9 protein targets closely related to different pharmacological applications described in Table IV. 
Predicted importance of ligand substructures
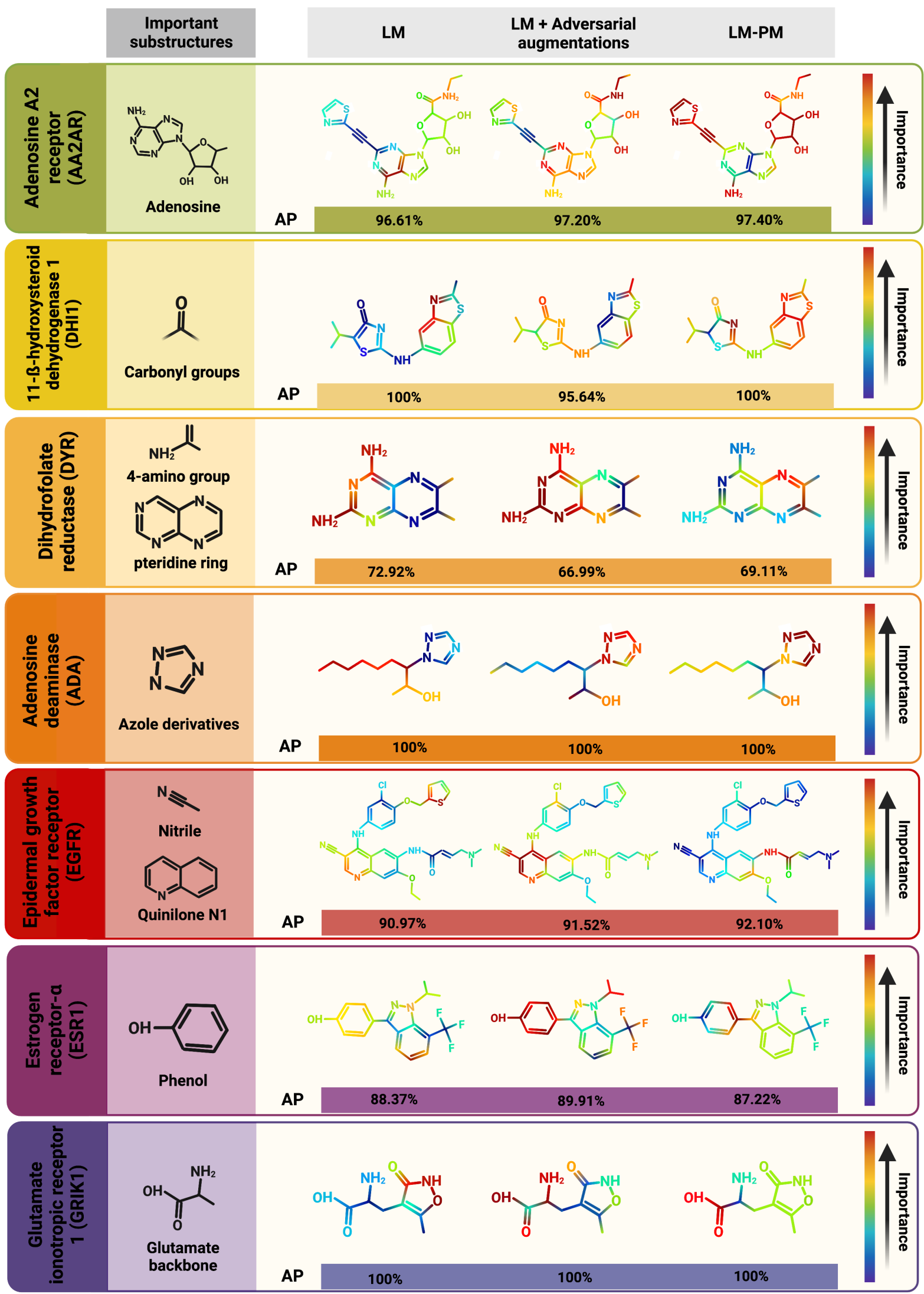

Fig. 7: Molecular salient feature maps. Importance of ligand substructures in PLIs predicted by the LM alone, the LM trained with adversarial augmentations, and the LM and PM jointly trained. Augmented models shift their focus towards substructures that best capture those previously reported to participate in PLIs. 


\section{Active compounds predicted for each target protein}


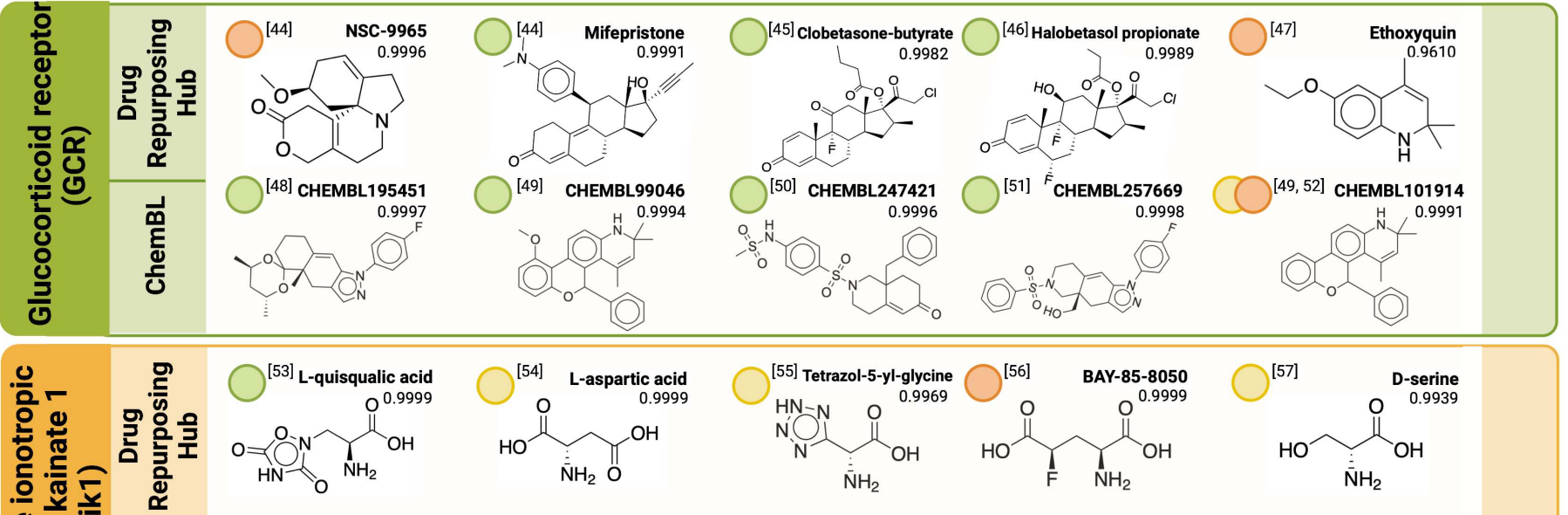

른

${ }^{[58]}$ CHEMBL337577

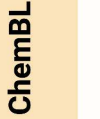

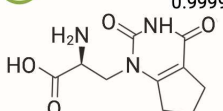

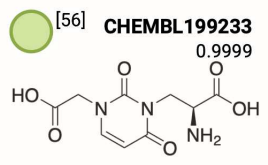

[59] CHEMBL283725

[60] CHEMBL3559410
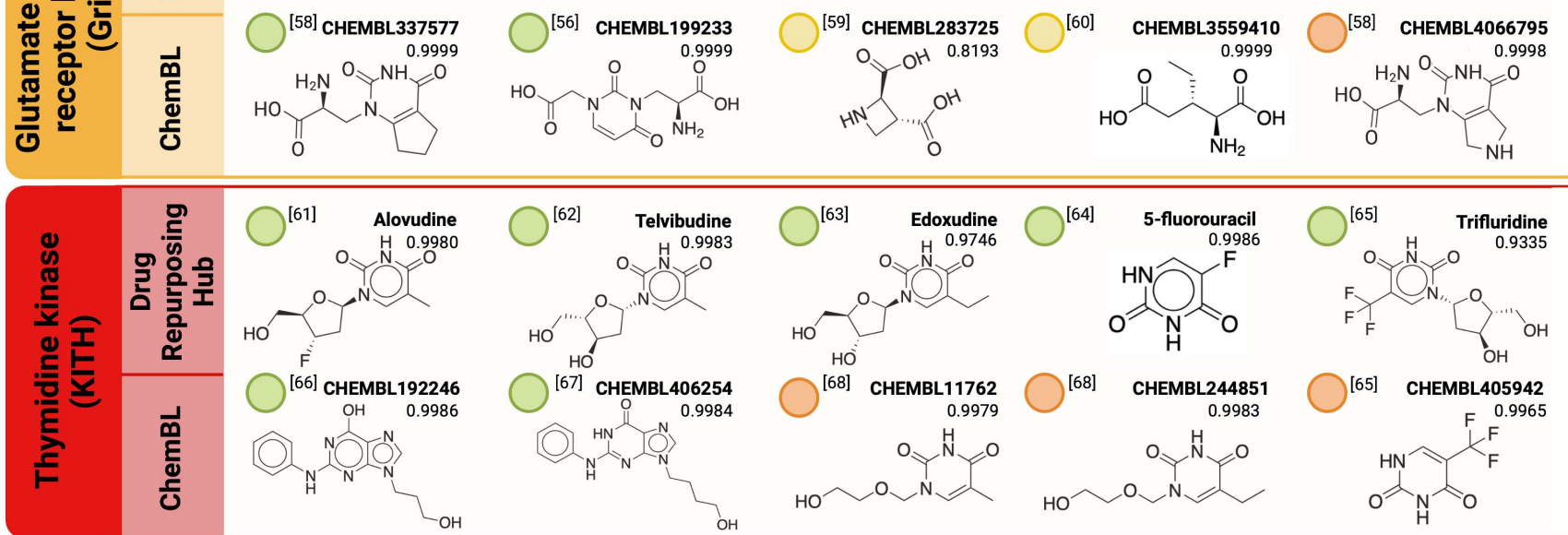

[68] CHEMBL11762 $\bigcirc$ [68] CHEMBL244851

(16)

$\mathrm{HO}_{\mathrm{NO}} \mathrm{N}$

[65] CHEMBL405942
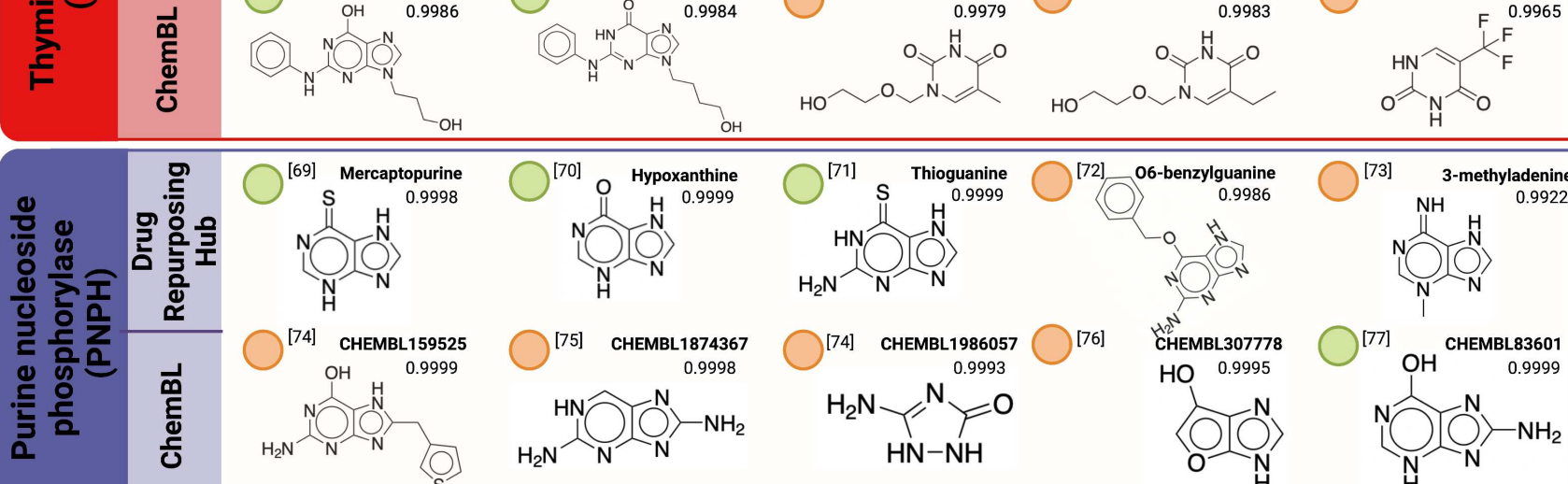

\section{[69] $\begin{array}{rr}\text { Mercaptopurine } \\ S & 0.9998\end{array}$ \\ 党}

[74] CHEMBL159525

$\mathrm{OH} 0.9999$

畓

$\mathrm{C}^{[78]}$ 3-deazaneplanocin-A



$\bigcirc^{[70]} \circ \begin{array}{r}\text { Hypoxanthine } \\ 0.9999\end{array}$<smiles>c1nnc2cn[nH]c2n1</smiles>

[75] CHEMBL1874367
0.9998

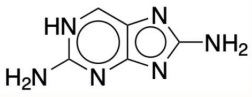

$\bigcirc^{[71]} s{ }^{\text {Thioguanine }}$

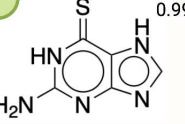

$\mathrm{H}_{2} \mathrm{~N} \overbrace{\mathrm{N}}$

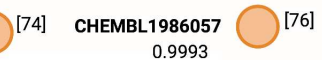

$\mathrm{H}_{2} \mathrm{~N} \underset{\mathrm{HN}-\mathrm{NH}}{\mathrm{N}}=0$

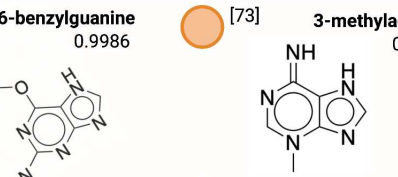

CHEMBL307778

HO 0.9995

$\widehat{O} \mathrm{O}_{\mathrm{H}}^{\mathrm{N}}$ $\int^{[77]} \mathrm{OH} \begin{array}{r}\text { CHEMBL83601 } \\ 0.9999\end{array}$

(O)

\section{$\bigcirc^{[34]} \mathrm{OH} \quad \begin{gathered}\text { Inositol } \\ 0.9996\end{gathered}$}

$\mathrm{HO}, \mathrm{OH}$

$\mathrm{OH}_{\mathrm{OH}}$

$\mathrm{OH}$

$\bigcirc^{[83]}$ CHEMBL50728

(1) $\bigcirc^{[79] \quad \begin{array}{c}\text { Tagatose } \\ 0.8844\end{array}}$

$\overbrace{}^{\mathrm{OH}} \mathrm{OH}_{\mathrm{OH}}$

ÖH OH

$\bigcirc^{[84]}$ CHEMBL76858

(1) $\int^{[79,80]}$ Dihydroxyacetone

[81] Cyclic AMP HO $\stackrel{\mathrm{O}}{\mathrm{OH}}$

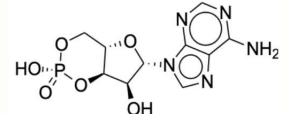

${ }^{\text {[85] }} \begin{array}{r}\text { CHEMBL610125 } \\ 0.9999\end{array}$

$\bigcirc^{[86]}$ CHEMBL405082

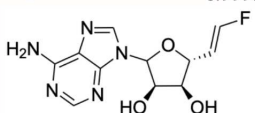






Fig. 8: PLA-Net's pharmacologically-relevant PLI predictions on the Drug Repurposing and CHEMBL databases. Five molecules from each database predicted as active with high probability are shown for nine pharmacologically-relevant targets. The name in the database and prediction probability for each molecule are shown on their upper right corner. Green label: experimentally-proven active molecule for the respective target. Yellow label: experimentally-proven active molecule for protein closely related to the target of interest. Orange label: not experimentally-proven, but with relevant substructures present in experimentally-proven active molecules for target.

Figure 8 shows five different molecules from each database that, with a high probability, are predicted to interact with each target. Green labeled molecules have been reported previously as active for the target of interest, yellow labeled molecules have been reported as active for a closely related protein, and finally, orange labeled molecules have not been reported but exhibit relevant substructures present in active molecules for the same target.

PLA-Net was able to accurately predict, for all target proteins, multiple molecules reported previously as active towards each target, as well as molecules that follow consistent structural patterns. All molecules predicted as active for farnesyl pyrophosphate synthase (FPPS), for example, have been reported as inhibitors due to the presence of biphosphate groups, which are thought to be implicated in their interaction with FPPS [41]. Similarly, most molecules predicted with activity towards PNPH, the purine nucleoside phosphatase, contain a purine substructure that correlates well with the
TABLE IV: Perfect-scoring targets with pharmacological applications used for virtual screening in the Drug Repourposing Hub and ChemBL databases.

\begin{tabular}{c|c|c}
\hline \hline Target protein & Acronym & Pharmacological Application \\
\hline \hline Adenosine deaminase & ADA & Immunomodulation \\
\hline Glucocorticoid receptor & GCR & Anti-inflammatory \\
\hline $\begin{array}{c}\text { Glutamate ionotropic } \\
\text { receptor kainate 1 }\end{array}$ & GRIK1 & Neurophysiological \\
\hline Thymidine kinase & KITH & Anticancer and Antimicrobial \\
\hline $\begin{array}{c}\text { C-X-C chemokine receptor } \\
\text { type 4 }\end{array}$ & CXCR4 & Antibacterial and Anticancer \\
\hline $\begin{array}{c}\text { Farnesyl pyrophosphase } \\
\text { synthase }\end{array}$ & FPPS & Anticancer and Antimicrobial \\
\hline $\begin{array}{c}\text { Purine nucleoside } \\
\text { phosphorylase }\end{array}$ & PNPH & Anticancer \\
\hline Adenosylhomocysteinase & SAHH & Anti-inflammatory \\
\hline Phospholipase A2 & PA2GA & Anticancer \\
\hline \hline
\end{tabular}

enzyme's function and, in turn, all molecules whose interaction 
has not been reported are either substructures of reported molecules or have slight ramification changes of known active molecules. This pattern was consistently observed with other targets such as the glucocorticoid receptor (GCR), the thymidine kinase (KITH) and the adenosylhomocysteinase (SAHH). In these cases, steroid backbones, thymine substructures and adenine substructures were present in most predicted ligands, respectively.

Despite these structural consistencies, some predicted molecules for targets such as SAHH, adenosine deaminase (ADA) and phospholipase A2 (PA2GA) showed no clear structural pattern, yet they still fit into one of two categories: (i) have been reported as active towards the protein or closely related proteins, or (ii) are substructures of active molecules for the target protein. In the particular case of SAHH, the predicted molecule Inositol has been experimentally proven as active towards this protein [42], but its structure largely differs from the other experimentally validated predictions. Similarly, the predicted active molecule for PA2GA, 1-hexadecanol, is a substructure of its experimentallyproven ligand 2-Ethylamino-1-hexadecanol [43], which fails to exhibit the purine-based structures observed previously in other experimentally-proven ligands for this target. The same trend is observed with the alcohol derivatives predicted for ADA, which are substructures of other previously reported ligands [44], [45] and their presence has shown to favor PLI occurrence [46]. This suggests that our model is able to learn relevant substructures from heterogeneous molecule sets and is not merely memorizing single structural patterns. Further evidence of this finding can be found by observing that several experimentally proven ligands for the $\mathrm{C}-\mathrm{X}-\mathrm{C}$ chemokine receptor type 4 (CXCR4) and the glutamate ionotropic receptor kianate 1 (Grik1) were accurately predicted, despite their marked structural differences and no apparent common backbone. Overall, these highly accurate predictions validate that PLANet is effectively learning underlying structural hallmarks that dictate PLIs with protein targets. This is of special clinical relevance given their close involvement in numerous diseases such as cancer, chronic inflammation, neurodegeneration, and microbial infections.

\section{CONCLUSIONS}

In this work, we propose PLA-Net, a two-module deep GCN to tackle PLI prediction in a curated version of the AD dataset. Our method merges rich information extracted from ligand and protein graphs utilizing deep GCN modules. Additionally, we propose a method for generating adversarial molecule augmentations that preserve biologically relevant backgrounds and show that their inclusion during training improves our model's performance and interpretability. Accordingly, PLA-Net not only becomes the new state-of-the-art in PLI prediction, but allows a more comprehensive analysis of the underlying features dictating PLIs. Moreover, its highly accurate PLI predictions with molecules unseen previously by the network and extracted from large and unannotated databases brings us one step closer towards interpretable pharmaceutical discovery.

\section{CODE AND DATA AVAILABILITY}

Code, implementation instructions, the curated AD dataset, PLA-Net weights and inference in drug repurposing and CHEMBL databases available at PLA-Net repository in https: //github.com/BCV-Uniandes/PLA-Net

\section{ACKNOWLEDGMENT}

This work was partially supported by a Microsoft AI for Health computational grant. The authors would also like to thank Juan Camilo Perez for his insights on adversarial data adaptation.

\section{REFERENCES}

[1] Wenqiang Cui, Adnane Aouidate, Shouguo Wang, Qiuliyang Yu, Yanhua Li, and Shuguang Yuan. Discovering anti-cancer drugs via computational methods. Frontiers in pharmacology, 11, 2020.

[2] Antonio Lavecchia and Carmen Cerchia. In silico methods to address polypharmacology: current status, applications and future perspectives. Drug Discovery Today, 21(2):288-298, 2016.

[3] David Thomas, Daniel Chancellor, Amanda Micklus, Sara LaFever, Michael Hay, Shomesh Chaudhuri, Robert Bowden, and Andrew W. Lo. Clinical Development Success Rates and Contributing Factors 2011-2020. 2021.

[4] The Food and Drug Administration. FDA Executive Summary. 2017.

[5] David C Swinney and Jason Anthony. How were new medicines discovered? Nature reviews Drug discovery, 10(7):507-519, 2011.

[6] Francesca Stanzione, Ilenia Giangreco, and Jason C Cole. Use of molecular docking computational tools in drug discovery. Progress in Medicinal Chemistry, 60:273-343, 2021.

[7] Sumudu P Leelananda and Steffen Lindert. Computational methods in drug discovery. Beilstein journal of organic chemistry, 12(1):26942718, 2016.

[8] Sharangdhar S Phatak, Clifford C Stephan, and Claudio N Cavasotto. High-throughput and in silico screenings in drug discovery. Expert Opinion on Drug Discovery, 4(9):947-959, 2009.

[9] Nicolaas M Angenent-Mari, Alexander S Garruss, Luis R Soenksen, George Church, and James J Collins. A deep learning approach to programmable rna switches. Nature communications, 11(1):1-12, 2020.

[10] Jacqueline A Valeri, Katherine M Collins, Pradeep Ramesh, Miguel A Alcantar, Bianca A Lepe, Timothy $\mathrm{K} \mathrm{Lu}$, and Diogo M Camacho. Sequence-to-function deep learning frameworks for engineered riboregulators. Nature communications, 11(1):1-14, 2020.

[11] Andrew W Senior, Richard Evans, John Jumper, James Kirkpatrick, Laurent Sifre, Tim Green, Chongli Qin, Augustin Žídek, Alexander WR Nelson, Alex Bridgland, et al. Improved protein structure prediction using potentials from deep learning. Nature, 577(7792):706-710, 2020.

[12] John Jumper, Richard Evans, Alexander Pritzel, Tim Green, Michae Figurnov, Olaf Ronneberger, Kathryn Tunyasuvunakool, Russ Bates, Augustin Žídek, Anna Potapenko, et al. Highly accurate protein structure prediction with alphafold. Nature, 596(7873):583-589, 2021.

[13] Nicolas Renaud, Cunliang Geng, Sonja Georgievska, Francesco Ambrosetti, Lars Ridder, Dario F Marzella, Manon F Réau, Alexandre MJJ Bonvin, and Li C Xue. Deeprank: A deep learning framework for data mining 3d protein-protein interfaces. Nature Communications, 12(1):1$8,2021$.

[14] Jonathan M Stokes, Kevin Yang, Kyle Swanson, Wengong Jin, Andres Cubillos-Ruiz, Nina M Donghia, Craig R MacNair, Shawn French, Lindsey A Carfrae, Zohar Bloom-Ackerman, et al. A deep learning approach to antibiotic discovery. Cell, 180(4):688-702, 2020.

[15] Benjamin Sanchez-Lengeling and Alán Aspuru-Guzik. Inverse molecular design using machine learning: Generative models for matter engineering. 361:360-365, 2018.

[16] Teva, Novartis, Mylan, Johnson Johnson, Pfizer, Bausch Health, GSK, ChemRar Group, Glenmark, Fujifilm, and et al. Global and China Drug Repositioning Market Size, Status and Forecast 2020-2027. QYResearch Group, OAD.

[17] Ahmet Sureyya Rifaioglu, Esra Nalbat, Volkan Atalay, Maria Jesus Martin, Rengul Cetin-Atalay, and Tunca Doğan. Deepscreen: high performance drug-target interaction prediction with convolutional neural networks using 2-d structural compound representations. Chemical science, 11(9):2531-2557, 2020. 
[18] Paola Ruiz Puentes, Natalia Valderrama, Cristina González, Laura Daza, Carolina Muñoz-Camargo, Juan C Cruz, and Pablo Arbeláez. Pharmanet: Pharmaceutical discovery with deep recurrent neural networks. bioRxiv, 2020.

[19] Jack Scantlebury, Nathan Brown, Frank Von Delft, and Charlotte M Deane. Data set augmentation allows deep learning-based virtual screening to better generalize to unseen target classes and highlight important binding interactions. Journal of Chemical Information and Modeling, 60(8):3722-3730, 2020.

[20] Zhirui Liao, Ronghui You, Xiaodi Huang, Xiaojun Yao, Tao Huang, and Shanfeng Zhu. Deepdock: Enhancing ligand-protein interaction prediction by a combination of ligand and structure information. In 2019 IEEE International Conference on Bioinformatics and Biomedicine (BIBM), pages 311-317. IEEE, 2019.

[21] Wen Torng and Russ B Altman. Graph convolutional neural networks for predicting drug-target interactions. Journal of chemical information and modeling, 59(10):4131-4149, 2019.

[22] Jaechang Lim, Seongok Ryu, Kyubyong Park, Yo Joong Choe, Jiyeon Ham, and Woo Youn Kim. Predicting drug-target interaction using a novel graph neural network with $3 \mathrm{~d}$ structure-embedded graph representation. Journal of chemical information and modeling, 59(9):39813988, 2019.

[23] Shuangjia Zheng, Yongjian Li, Sheng Chen, Jun $\mathrm{Xu}$, and Yuedong Yang. Predicting drug-protein interaction using quasi-visual question answering system. Nature Machine Intelligence, 2(2):134-140, 2020.

[24] Guohao Li, Matthias Müller, Ali Thabet, and Bernard Ghanem. Deepgcns: Can gens go as deep as cnns? In The IEEE International Conference on Computer Vision (ICCV), 2019.

[25] Guohao Li, Chenxin Xiong, Ali Thabet, and Bernard Ghanem. Deepergen: All you need to train deeper gens. arXiv, 2020.

[26] Hanjun Dai, Hui Li, Tian Tian, Xin Huang, Lin Wang, Jun Zhu, and Le Song. Adversarial attack on graph structured data. In International conference on machine learning, pages 1115-1124. PMLR, 2018.

[27] Chembl. The Brussels Times.

[28] Steven M Corsello, Joshua A Bittker, Zihan Liu, Joshua Gould, Patrick McCarren, Jodi E Hirschman, Stephen E Johnston, Anita Vrcic, Bang Wong, Mariya Khan, et al. The drug repurposing hub: a next-generation drug library and information resource. Nature medicine, 23(4):405408, 2017.

[29] John. J. Irwin Michael M. Mysinger, Michael Carchia and Brian K. Shoichet. Directory of useful decoys, enhanced (dud-e): Better ligands and decoys for better benchmarking. Journal of Medicinal Chemistry, 55:6582-6594, 2012.

[30] Ann E Cleves and Ajay N Jain. Structure-and ligand-based virtual screening on dud-e+: Performance dependence on approximations to the binding pocket. Journal of chemical information and modeling, 60(9):4296-4310, 2020.

[31] Ludovic Chaput, Juan Martinez-Sanz, Nicolas Saettel, and Liliane Mouawad. Benchmark of four popular virtual screening programs: construction of the active/decoy dataset remains a major determinant of measured performance. Journal of cheminformatics, 8(1):1-17, 2016.

[32] Lieyang Chen, Anthony Cruz, Steven Ramsey, Callum J Dickson, Jose S Duca, Viktor Hornak, David R Koes, and Tom Kurtzman. Hidden bias in the dud-e dataset leads to misleading performance of deep learning in structure-based virtual screening. PloS one, 14(8), 2019.

[33] F. Yu and V. Koltun. Multi-scale context aggregation by dilated convolutions. CoRR, abs/1511.07122, 2016.

[34] Guy W Bemis and Mark A Murcko. The properties of known drugs. 1. molecular frameworks. Journal of medicinal chemistry, 39(15):28872893, 1996.

[35] Guillaume Lebon Byron Carpenter. Human adenosine a2a receptor: Molecular mechanism of ligand binding and activation. Frontiers in Pharmacology, 8, 2017.

[36] Rosaria Volpini Sauro Vittori Emido Camaioni Gloria Cristalli, Alessandra Eleuteri and Giulio Lupidi. Adenosine deaminase inhibitors: synthesis and structure-activity relationships of 2-hydroxy-3nonyl derivatives of azoles. Journal of Medicinal Chemistry, 37, 1994

[37] Stephen J. Benkovic Kazunari Taira. Evaluation of the importance of hydrophobic interactions in drug binding to dihydrofolate reductase. Journal of Medicinal Chemistry, 31, 1988.

[38] William A. Hallett Marvin F. Reich M. Brawner Floyd Bernard D. Johnson Ronald S. Michalak Ramaswamy Nilakantan Carolyn Discafani Jonathan Golas Sridhar K. Rabindran Ru Shen Xiaoqing Shi Yu-Fen Wang Janis Upeslacis Allan Wissner Hwei-Ru Tsou, Elsebe G. Overbeek-Klumpers. Optimization of 6,7-disubstituted-4(arylamino)quinoline-3-carbonitriles as orally active, irreversible in- hibitors of human epidermal growth factor receptor-2 kinase activity. Journal of Medicinal Chemistry, 48, 2005.

[39] Friedrich C. Luft. 11-hydroxysteroid dehydrogenase-2 and saltsensitive hypertension. Circulation, 133, 2016.

[40] Mark A Ashwell William J Moore William R Solvibile Eugene Trybulski Christopher C Chadwick Susan Chippari Thomas Kenney Amy Eckert Lisa Borges-Marcucci James C Keith Zhang Xu Lydia Mosyak Douglas C Harnish Robert J Steffan, Edward Matelan. Synthesis and activity of substituted 4-(indazol-3-yl)phenols as pathway-selective estrogen receptor ligands useful in the treatment of rheumatoid arthritis. Journal of Medicinal Chemistry, 47, 2004.

[41] K Ohno, Kenichi Mori, M Orita, and M Takeuchi. Computational insights into binding of bisphosphates to farnesyl pyrophosphate synthase. Current medicinal chemistry, 18(2):220-233, 2011.

[42] Heimo Wolinski Gerald Rechberger Sepp D.Kohlwein Oksana Tehlivets Nermina Malanovic, Ingo Streith. S-adenosyl1-homocysteine hydrolase, key enzyme of methylation metabolism, regulates phosphatidylcholine synthesis and triacylglycerol homeostasis in yeast: Implications for homocysteine as a risk factor of atherosclerosis. Journal of Biological Chemistry, 283, 2008.

[43] M. Payá M. Alves E. del Olmo JL. López A. San Feliciano R. Lucas A. Ubeda. Synthesis and enzyme inhibitory activities of a series of lipidic diamine and aminoalcohol derivatives on cytosolic and secretory phospholipases a2. Bioorganic and Medicinal Chemistry Letters, 10, 2000.

[44] Lars Oliver Conzelmann Harald Winkler Stefan Hofer Jochen Steppan Heinfried Schmidt Hubert Bardenheuer Christian-Friedrich Vahl Markus A Weigand Nalan Kayhan, Benjamin Funke. The adenosine deaminase inhibitor erythro-9-[2-hydroxyl-3-nonyl]-adenine decreases intestinal permeability and protects against experimental sepsis: a prospective, randomised laboratory investigation. Critical Care, 12, 2008.

[45] P. Serafinowski R. McKenna, S. Neidle. Structure of 5'-chloro-3',5'dideoxyformycin a monohydrate. the effects of protonation on formycin structure and conformation. Acta Crystallographica, 46, 1990.

[46] Ralph R. Rossi Leon M. Lerner. Inhibition of adenosine deaminase by alcohols derived from adenine nucleosides. Biochemistry, 11, 1972.

[47] John M. Sanders, Yongcheng Song, Julian M. W. Chan, Yonghui Zhang, Samuel Jennings, Thomas Kosztowski, Sarah Odeh, Ryan Flessner, Christine Schwerdtfeger, Evangelia Kotsikorou, Gary A. Meints, Aurora Ortiz Gómez, Dolores González-Pacanowska, Amy M Raker, Hong Wang, Ermond R. van Beek, Socrates E. Papapoulos, Craig T. Morita, and Eric Oldfield. Pyridinium-1-yl bisphosphonates are potent inhibitors of farnesyl diphosphate synthase and bone resorption. Journal of Medicinal Chemistry, 48(8):2957-2963, April 2005.

[48] John M. Sanders, Aurora Ortiz Gómez, Junhong Mao, Gary A. Meints, Erin M. Van Brussel, Agnieszka Burzynska, Pawel Kafarski, Dolores González-Pacanowska, and Eric Oldfield. 3-d QSAR investigations of the inhibition of leishmania major farnesyl pyrophosphate synthase by bisphosphonates. Journal of Medicinal Chemistry, 46(24):5171-5183, October 2003.

[49] Sergio H. Szajnman, Guadalupe E. Garcı'a Liñares, Zhu-Hong Li, Cuiying Jiang, Melina Galizzi, Esteban J. Bontempi, Marcela Ferella, Silvia N.J. Moreno, Roberto Docampo, and Juan B. Rodriguez. Synthesis and biological evaluation of 2-alkylaminoethyl-1, 1-bisphosphonic acids against trypanosoma cruzi and toxoplasma gondii targeting farnesyl diphosphate synthase. Bioorganic \& Medicinal Chemistry, 16(6):3283-3290, March 2008.

[50] Daniele Simoni, Nicola Gebbia, Francesco Paolo Invidiata, Marco Eleopra, Paolo Marchetti, Riccardo Rondanin, Riccardo Baruchello, Stefano Provera, Carla Marchioro, Manlio Tolomeo, Luciana Marinelli, Vittorio Limongelli, Ettore Novellino, Aaron Kwaasi, James Dunford, Simona Buccheri, Nadia Caccamo, and Francesco Dieli. Design, synthesis, and biological evaluation of novel aminobisphosphonates possessing an in vivo antitumor activity through a y-t lymphocytesmediated activation mechanism. Journal of Medicinal Chemistry, 51(21):6800-6807, October 2008.

[51] Evangelia Kotsikorou and Eric Oldfield. A quantitative structureactivity relationship and pharmacophore modeling investigation of aryl$\mathrm{x}$ and heterocyclic bisphosphonates as bone resorption agents. Journal of Medicinal Chemistry, 46(14):2932-2944, July 2003.

[52] Frederic Castinetti, Bernard Conte-Devolx, and Thierry Brue. Medical treatment of cushing's syndrome: Glucocorticoid receptor antagonists and mifepristone. Neuroendocrinology, 92(1):125-130, 2010.

[53] D D Munro and L Wilson. Clobetasone butyrate, a new topical corticosteroid: clinical activity and effects on pituitary-adrenal axis 
function and model of epidermal atrophy. BMJ, 3(5984):626-628, September 1975.

[54] Halobetasol propionate. Website, 2021.

[55] Alberta L. Wang, Ronald Panganiban, Weiliang Qiu, Alvin T. Kho, Geoffrey Chupp, Deborah A. Meyers, Eugene R. Bleecker, Scott T. Weiss, Quan Lu, and Kelan G. Tantisira. Drug repurposing to treat glucocorticoid resistance in asthma. Journal of Personalized Medicine, 11(3): 175, March 2021.

[56] Process and intermediates for the synthesis of 8-[1-(3,5bis-(trifluoromethyl)phenyl)-ethoxy-methyl]-8-phenyl-1,7-diazaspiro[4.5]decan-2-one compounds. Website, 2021.

[57] Michael J. Coghlan, Philip R. Kym, Steven W. Elmore, Alan X Wang, Jay R. Luly, Denise Wilcox, Michael Stashko, Chun-Wei Lin, Jeffrey Miner, Curtis Tyree, Masaki Nakane, Peer Jacobson, and Benjamin C. Lane. Synthesis and characterization of non-steroidal ligands for the glucocorticoid receptor: selective quinoline derivatives with prednisolone-equivalent functional activity. Journal of Medicinal Chemistry, 44(18):2879-2885, August 2001.

[58] Robin D. Clark, Nicholas C. Ray, Paul Blaney, Peter H. Crackett, Christopher Hurley, Karen Williams, Hazel J. Dyke, David E. Clark, Peter M. Lockey, Rene Devos, Melanie Wong, Anne White, and Joseph K. Belanoff. 2-benzenesulfonyl-8a-benzyl-hexahydro-2hisoquinolin-6-ones as selective glucocorticoid receptor antagonists. Bioorganic \& Medicinal Chemistry Letters, 17(20):5704-5708, October 2007.

[59] Robin D. Clark, Nicholas C. Ray, Karen Williams, Paul Blaney, Stuart Ward, Peter H. Crackett, Christopher Hurley, Hazel J. Dyke, David E. Clark, Peter Lockey, Rene Devos, Melanie Wong, Soraya S. Porres, Colin P. Bright, Robert E. Jenkins, and Joseph Belanoff. 1h-pyrazolo[3, 4-g]hexahydro-isoquinolines as selective glucocorticoid receptor antagonists with high functional activity. Bioorganic \& Medicinal Chemistry Letters, 18(4):1312-1317, February 2008.

[60] Christopher M. Tegley, Lin Zhi, Keith B. Marschke, Marco M. Gottardis, Qinchuan Yang, and Todd K. Jones. 5-benzylidene 1, 2-dihydrochromeno[3, 4-f]quinolines, a novel class of nonsteroidal human progesterone receptor agonists. Journal of Medicinal Chemistry, 41(22):4354-4359, September 1998.

[61] William A. Kinney, Nancy E. Lee, Deanna T. Garrison, Edward J. Podlesny, Joanne T. Simmonds, Donna Bramlett, Ronald R. Notvest, Dianne M. Kowal, and Rene P. Tasse. Bioisosteric replacement of the alpha.-amino carboxylic acid functionality in 2-amino-5phosphonopentanoic acid yields unique 3, 4-diamino-3-cyclobutene1, 2-dione containing NMDA antagonists. Journal of Medicinal Chemistry, 35(25):4720-4726, December 1992.

[62] Yanhua H. Huang, Saurabh R. Sinha, Olesya D. Fedoryak, Graham C. R. Ellis-Davies, and Dwight E. Bergles. Synthesis and characterization of 4-methoxy-7-nitroindolinyl-d-aspartate, a caged compound for selective activation of glutamate transporters andN-methyl-d-aspartate receptors in brain tissue $\dagger$. Biochemistry, 44(9):3316-3326, March 2005.

[63] Darryle D. Schoepp, Craig L. Smith, David Lodge, John D. Millar, J.David Leander, Aida I. Sacaan, and William H.W. Lunn. D, 1(tetrazol-5-yl) glycine: a novel and highly potent NMDA receptor agonist. European Journal of Pharmacology, 203(2):237-243, October 1991.

[64] Nigel P. Dolman, Helen M. Troop, Julia C. A. More, Andrew Alt, Jody L. Knauss, Robert Nistico, Samantha Jack, Richard M. Morley, Zuner A. Bortolotto, Peter J. Roberts, David Bleakman, Graham L. Collingridge, and David E. Jane. Synthesis and pharmacology of willardiine derivatives acting as antagonists of kainate receptors. Journal of Medicinal Chemistry, 48(24):7867-7881, December 2005.

[65] Alfred C. Chin, Remy A. Yovanno, Tyler J. Wied, Ariel Gershman, and Albert Y. Lau. D-serine potently drives ligand-binding domain closure in the ionotropic glutamate receptor GluD2. Structure, 28(10):11681178.e2, October 2020.

[66] Stefania Butini, Darryl S. Pickering, Elena Morelli, Salvatore Sanna Coccone, Francesco Trotta, Meri De Angelis, Egeria Guarino, Isabella Fiorini, Giuseppe Campiani, Ettore Novellino, Arne Schousboe, Jeppe K. Christensen, and Sandra Gemma. 1h-cyclopentapyrimidine$2,4(1 \mathrm{~h}, 3 \mathrm{~h})$-dione-related ionotropic glutamate receptors ligands. structure-activity relationships and identification of potent and selective iGluR5 modulators. Journal of Medicinal Chemistry, 51(20):6614 6618, October 2008.

[67] Elżbieta Ziemińska, Aleksandra Stafiej, and Jerzy W Łazarewicz. Role of group i metabotropic glutamate receptors and NMDA receptors in homocysteine-evoked acute neurodegeneration of cultured cerebellar granule neurones. Neurochemistry International, 43(4-5):481-492, September 2003.

[68] Alan P. Kozikowski, Werner Tuckmantel, Ian J. Reynolds, and Jarda T. Wroblewski. Synthesis and bioactivity of a new class of rigid glutamate analogs. modulators of the n-methyl-d-aspartate receptor. Journal of Medicinal Chemistry, 33(6):1561-1571, June 1990.

[69] Morse Faria, Matthew S. Halquist, Erick Kindt, Wenlin Li, H. Thomas Karnes, and Peter J. O'Brien. Liquid chromatography-tandem mass spectrometry method for quantification of thymidine kinase activity in human serum by monitoring the conversion of 3/-deoxy-3/ fluorothymidine to 3/-deoxy-3/-fluorothymidine monophosphate. Journal of Chromatography B, 907:13-20, October 2012.

[70] Edward G. Bridges, Jules R. Selden, and Shouqi Luo. Nonclinica safety profile of telbivudine, a novel potent antiviral agent for treatment of hepatitis b. Antimicrobial Agents and Chemotherapy, 52(7):25212528, July 2008 .

[71] T. Kulikowski. Structure-activity relationships and conformational features of antiherpetic pyrimidine and purine nucleoside analogues. a review. Pharmacy World \& Science, 16(2):127-138, April 1994.

[72] Youhoon Chong and Chung K Chu. Understanding the unique mechanism of 1-FMAU (clevudine) against hepatitis b virus: molecular dynamics studies. Bioorganic \& Medicinal Chemistry Letters, 12(23):3459-3462, December 2002.

[73] Norihiko Suzuki. Mode of action of trifluorothymidine (TFT) against DNA replication and repair enzymes. International Journal of Oncology, April 2011.

[74] Andrzej Manikowski, Annalisa Verri, Andrea Lossani, Bryan M. Geb hardt, Joseph Gambino, Federico Focher, Silvio Spadari, and George E. Wright. Inhibition of herpes simplex virus thymidine kinases by 2 phenylamino-6-oxopurines and related compounds: structure-activity relationships and antiherpetic activity in vivo. Journal of Medicinal Chemistry, 48(11):3919-3929, June 2005.

[75] Hongyan Xu, Giovanni Maga, Federico Focher, Emil R. Smith, Silvio Spadari, Joseph Gambino, and George E. Wright. Synthesis, properties, and pharmacokinetic studies of n2-phenylguanine derivatives as inhibitors of herpes simplex virus thymidine kinases. Journal of Medicinal Chemistry, 38(1):49-57, January 1995.

[76] J. Balzarini, C. Bohman, and E. De Clercq. Differential mechanism of cytostatic effect of (e)-5-(2-bromovinyl)-2'-deoxyuridine, 9-(1, 3dihydroxy-2-propoxymethyl)guanine, and other antiherpetic drugs on tumor cells transfected by the thymidine kinase gene of herpes simplex virus type 1 or type 2. Journal of Biological Chemistry, 268(9):63326337, March 1993.

[77] T A Krenitsky, G B Elion, A M Henderson, and G H Hitchings. Inhibition of human purine nucleoside phosphorylase. Journal of Biological Chemistry, 243(11):2876-2881, June 1968.

[78] Gertraud Koellner, Marija Luić, David Shugar, Wolfram Saenger, and Agnieszka Bzowska. Crystal structure of calf spleen purine nucleoside phosphorylase in a complex with hypoxanthine at 2.15 a a resolution. Journal of Molecular Biology, 265(2):202-216, January 1997.

[79] Sang He Lee and Alan C. Sartorelli. Conversion of 6-thioguanine to the nucleoside level by purine nucleoside phosphorylase of sarcoma 180 and sarcoma 180/tg ascites cells. CANCER RESEARCH, 41:10861090, 1981.

[80] An enzymatic synthesis of nucleosides of n2-acetyl-o6-[2-(4nitrophenyl)ethyl]guanine. Website, 2021.

[81] Agnieszka Bzowska, Ewa Kulikowska, and David Shugar. Linear free energy relationships for $\mathrm{n}(7)$-substituted guanosines as substrates of calf spleen purine nucleoside phosphorylase. possible role of $\mathrm{n}(7)$ protonation as an intermediary in phosphorolysis. Zeitschrift für Naturforschung C, 48(9-10):803-811, October 1993.

[82] Taras Chaban1, Olena Klenina, Ihor Chaban, Volodymyr Ogurtsov, Stefan Harkov, and Maryan Lelyukh. Thiazolo[5,4-d]pyrimidines and thiazolo[4,5-d] pyrimidines: Review on synthesis and pharmacological importance of their derivatives. PHARMACIA, 65:54-70, 2018.

[83] Gertraud Koellner, Albrecht Stroh, Grzegorz Raszewski, Antonin Holý, and Agnieszka Bzowska. Crystal structure of calf spleen purine nucleoside phosphorylase in a complex with multisubstrate analogue inhibitor with 2, 6-diaminopurine aglycone. Nucleosides, Nucleotides and Nucleic Acids, 22(5-8):1699-1702, October 2003.

[84] Claudia López-Lira, Ricardo A. Tapia, Alejandra Herrera, Miche Lapier, Juan D. Maya, Jorge Soto-Delgado, Allen G. Oliver, A. Graham Lappin, and Eugenio Uriarte. New benzimidazolequinones as trypanosomicidal agents. Bioorganic Chemistry, 111:104823, June 2021.

[85] Johanna D. Stoeckler, Carolyn Cambor, Valerie Kuhns, Chu Shih-Hsi, and Robert E. Parks. Inhibitors of purine nucleoside phosphorylase. Biochemical Pharmacology, 31(2):163-171, January 1982. 
[86] Juliette Aury-Landas, Céline Bazille, Lyess Allas, Sara Bouhout, Christophe Chesneau, Sylvain Leclercq, Karim Boumédiene, and Catherine Baugé. Anti-inflammatory and chondroprotective effects of the s-adenosylhomocysteine hydrolase inhibitor 3-deazaneplanocin a, in human articular chondrocytes. Scientific Reports, 7(1), July 2017.

[87] Keyong Yuan, Lili Hou, Qiaoqiao Jin, Chenguang Niu, Mengying Mao, Ruirui Wang, and Zhengwei Huang. Comparative transcriptomics analysis of streptococcus mutans with disruption of LuxS/AI-2 quorum sensing and recovery of methyl cycle. Archives of Oral Biology, 127:105137, July 2021.

[88] Venkata L.A. Malladi, Adam J. Sobczak, Tiffany M. Meyer, Dehua Pei, and Stanislaw F. Wnuk. Inhibition of LuxS by s-ribosylhomocysteine analogues containing a [4-aza]ribose ring. Bioorganic \& Medicinal Chemistry, 19(18):5507-5519, September 2011.

[89] Per Magne Ueland and John Saebo. S-adenosylhomocysteinase from mouse liver. effect of adenine and adenine nucleotides on the enzyme catalysis. Biochemistry, 18(19):4130-4135, September 1979.

[90] David R. Borcherding, Sunanda Narayanan, Masahide Hasobe, James G. McKee, Bradley T. Keller, and Ronald T. Borchardt. Potential inhibitors of s-adenosylmethionine-dependent methyltransferases. 11. molecular dissections of neplanocin a as potential inhibitors of sadenosylhomocysteine hydrolase. Journal of Medicinal Chemistry, 31(9):1729-1738, September 1988.

[91] Michael S. Wolfe, Younha Lee, William J. Bartlett, David R. Borcherding, and Ronald T. Borchardt. 4'-modified analogs of aristeromycin and neplanocin a: synthesis and inhibitory activity toward s-adenosyl-1-homocysteine hydrolase. Journal of Medicinal Chemistry, 35(10):1782-1791, May 1992.

[92] Siming Liu, Chong sheng Yuan, and Ronald T. Borchardt Aristeromycin-5'-carboxaldehyde: a potent inhibitor of s-adenosyl1-homocysteine hydrolase. Journal of Medicinal Chemistry, 39(12):2347-2353, January 1996.

[93] Stanislaw F. Wnuk, Chong-Sheng Yuan, Ronald T. Borchardt, Jan Balzarini, Erik De Clercq, and Morris J. Robins. Nucleic acid-related compounds. 84. synthesis of 6'-(e and $\mathrm{z}$ )-halohomovinyl derivatives of adenosine, inactivation of s-adenosyl-1-homocysteine hydrolase, and correlation of anticancer and antiviral potencies with enzyme inhibition. Journal of Medicinal Chemistry, 37(21):3579-3587, October 1994.

[94] Takayuki Ando, Kenji Kojima, Praveen Chahota, Atsushi Kozaki, Nikalje D. Milind, and Yukio Kitade. Synthesis of 4/-modified noraristeromycins to clarify the effect of the 4/-hydroxyl groups for inhibitory activity against s-adenosyl-1-homocysteine hydrolase. Bioorganic \& Medicinal Chemistry Letters, 18(8):2615-2618, April 2008.

[95] Varnavas D. Mouchlis, Aaron Armando, and Edward A. Dennis. Substrate-specific inhibition constants for phospholipase a2 acting on unique phospholipid substrates in mixed micelles and membranes using lipidomics. Journal of Medicinal Chemistry, 62(4):1999-2007, January 2019.

[96] Robert D. Dillard, Nicholas J. Bach, Susan E. Draheim, Dennis R. Berry, Donald G. Carlson, Nickolay Y. Chirgadze, David K. Clawson, Lawrence W. Hartley, Lea M. Johnson, Noel D. Jones, Emma R. McKinney, Edward D. Mihelich, Jennifer L. Olkowski, Richard W. Schevitz, Amy C. Smith, David W. Snyder, Cynthia D. Sommers, and Jean-Pierre Wery. Indole inhibitors of human nonpancreatic secretory phospholipase a2. 2. indole-3-acetamides with additional functionality. Journal of Medicinal Chemistry, 39(26):5137-5158, January 1996.

[97] Aid 720700 - fluorescence-based biochemical high throughput primary assay to identify inhibitors of phospholipase c isozymes (plc-gamma1). - pubchem. National Center for Biotechnology Information, 2021.

[98] Brian P. Smart, Rob C. Oslund, Laura A. Walsh, and Michael H. Gelb. The first potent inhibitor of mammalian group x secreted phospholipase a2: elucidation of sites for enhanced binding. Journal of Medicinal Chemistry, 49(10):2858-2860, April 2006.

[99] Ilze Adlere, Birgit Caspar, Marta Arimont, Sebastian Dekkers, Kirsten Visser, Jeffrey Stuijt, Chris de Graaf, Michael Stocks, Barrie Kellam, Stephen Briddon, Maikel Wijtmans, Iwan de Esch, Stephen Hill, and Rob Leurs. Modulators of CXCR4 and CXCR7/ACKR3 function. Molecular Pharmacology, 96(6):737-752, September 2019.

[100] Eric M. Rosenberg, Reed E.S. Harrison, Lun Kelvin Tsou, Natalie Drucker, Brock Humphries, Deepa Rajasekaran, Kathryn E. Luker, Chien-Huang Wu, Jen-Shin Song, Chuan-Jen Wang, James W. Murphy, Yung-Chi Cheng, Kak-Shan Shia, Gary D. Luker, Dimitrios Morikis, and Elias J. Lolis. Characterization, dynamics, and mechanism of CXCR4 antagonists on a constitutively active mutant. Cell Chemical Biology, 26(5):662-673.e7, May 2019.

[101] Victoria A. Johnson, Yoninah S. Cramer, Susan L. Rosenkranz, Stephen Becker, Karin L. Klingman, Beatrice Kallungal, Eoin Coakley, Ed- ward P. Acosta, Gary Calandra, Michael S. Saag, Roger Bedimo, Susan Owens, Elaine Ferguson, Lisa Kessels, David Shugarts, Vincent Parrillo, Kerry Upton, V. White, Mitchell Goldman, W. Zwickl, Carlos del Rio, Akaki Turkia, Amanda Zadzilk, J. Darren Hazelwood, and Darlene $\mathrm{Lu}$ and. Antiretroviral activity of AMD11070 (an orally administered CXCR4 entry inhibitor): Results of NIH/NIAID AIDS clinical trials group protocol a5210. AIDS Research and Human Retroviruses, 35(8):691-697, August 2019.

[102] Astrid S. Jørgensen, Viktorija Daugvilaite, Katia De Filippo, Christian Berg, Masa Mavri, Tau Benned-Jensen, Goda Juzenaite, Gertrud Hjortø, Sara Rankin, Jon Våben $\varnothing$, and Mette M. Rosenkilde. Biased action of the CXCR4-targeting drug plerixafor is essential for its superior hematopoietic stem cell mobilization. Communications Biology, 4(1), May 2021.

[103] Royce A. Wilkinson, Seth H. Pincus, Kejing Song, Joyce B. Shepard, Alan J. Weaver, Mohamed E. Labib, and Martin Teintze. Improved guanide compounds which bind the CXCR4 co-receptor and inhibit HIV-1 infection. Bioorganic \& Medicinal Chemistry Letters, 23(7):2197-2201, April 2013.

[104] Gebhard Thoma, Markus B. Streiff, Jiri Kovarik, Fraser Glickman, Trixie Wagner, Christian Beerli, and Hans-Günter Zerwes. Orally bioavailable isothioureas block function of the chemokine receptor CXCR4 in vitro and in vivo. Journal of Medicinal Chemistry, 51(24):7915-7920, November 2008.

[105] Renato Skerlj, Gary Bridger, Ernie McEachern, Curtis Harwig, Chris Smith, Trevor Wilson, Duane Veale, Helen Yee, Jason Crawford, Krystyna Skupinska, Rossana Wauthy, Wen Yang, Yongbao Zhu, David Bogucki, Maria Di Fluri, Jonathon Langille, Dana Huskens, Erik De Clercq, and Dominique Schols. Synthesis and SAR of novel CXCR4 antagonists that are potent inhibitors of $t$ tropic (x4) HIV-1 replication. Bioorganic \& Medicinal Chemistry Letters, 21(1):262-266, January 2011.

[106] Saibal Chakraborty, Niti H. Shah, James C. Fishbein, and Ramachandra S. Hosmane. Investigations into specificity of azepinomycin for inhibition of guanase: Discrimination between the natural heterocyclic inhibitor and its synthetic nucleoside analogues. Bioorganic \& Medic inal Chemistry Letters, 22(23):7214-7218, December 2012.

[107] Srinivas Rao Kasibhatla, Brett C. Bookser, Gary Probst, James R. Appleman, and Mark D. Erion. AMP deaminase inhibitors. 3. SAR of 3-(carboxyarylalkyl)coformycin aglycon analogues. Journal of Medicinal Chemistry, 43(8):1508-1518, March 2000.

[108] Brett C. Bookser, Srinivas Rao Kasibhatla, James R. Appleman, and Mark D. Erion. AMP deaminase inhibitors. 2. initial discovery of a non-nucleotide transition-state inhibitor series. Journal of Medicinal Chemistry, 43(8):1495-1507, March 2000. 